\title{
Transatlantica
}

Revue d'études américaines. American Studies Journal

$2 \mid 2011$

Sport et société / Animals and the American

Imagination

\section{Democracy Promotion and Nation Building in United States Foreign Policy}

Part I: Democracy from America? Historical Continuities in U.S. Nation Building Policies

\section{Myriam Chasserieau}

\section{(2) OpenEdition}

\section{Journals}

Édition électronique

URL : https://journals.openedition.org/transatlantica/5686

DOI : $10.4000 /$ transatlantica.5686

ISSN : $1765-2766$

Éditeur

Association française d'Etudes Américaines (AFEA)

Référence électronique

Myriam Chasserieau, "Democracy Promotion and Nation Building in United States Foreign Policy », Transatlantica [En ligne], 2 | 2011, mis en ligne le 17 juin 2012, consulté le 01 février 2023. URL : http:// journals.openedition.org/transatlantica/5686; DOI : https://doi.org/10.4000/transatlantica.5686

Ce document a été généré automatiquement le 1 février 2023

Creative Commons - Attribution - Pas d'Utilisation Commerciale - Pas de Modification 4.0 International - CC BY-NC-ND 4.0

https://creativecommons.org/licenses/by-nc-nd/4.0/ 


\section{Democracy Promotion and Nation Building in United States Foreign Policy}

Part I: Democracy from America? Historical Continuities in U.S. Nation Building Policies

Myriam Chasserieau

1 La journée d'études internationale «Democracy Promotion and Nation Building in United States Foreign Policy, Part I: Democracy from America? Historical Continuities in U.S. Nation Building Policies » organisée le 15 octobre 2011 par Annick CIZEL et Alexandra de HOOP SCHEFFER dans le cadre des activités de l'Observatoire de la Politique Américaine du Center for Research in the English-Speaking World (CREW - EA 4399) de l'Université Sorbonne Nouvelle - Paris 3, a fait dialoguer des chercheurs européens et américains sur le concept de nation building et sur le paradigme fondateur des États-Unis qu'est la promotion de la démocratie. Historiens et politologues y ont proposé une réflexion sur les continuités et les évolutions des stratégies d'exportation de la démocratie. Bien que les concepts de nation building et de democracy promotion soient relativement récents, ils sont, depuis la fondation de la République, au cœur de la politique étrangère américaine. Les États-Unis se sont en effet posés en modèle de démocratie pour les autres peuples dès l'époque des Pères fondateurs, à l'image de Thomas Jefferson, promoteur d'un « Empire de la Liberté » sur le continent.

2 Lors de la première session dédiée à l'affirmation de l'exceptionnalisme américain au berceau de la promotion de la démocratie durant la première moitié du vingtième siècle, Serge RICARD (Université Sorbonne Nouvelle - Paris 3) insiste sur l'influence du président Theodore Roosevelt sur la politique étrangère des États-Unis au xxème siècle. Les historiens ont trop souvent associé l'émergence des États-Unis sur la scène politique internationale à la présidence de Woodrow Wilson dont l'idéologie aurait conféré au modèle américain de démocratie libérale une supériorité morale. Cependant, rappelle Serge Ricard, c'est sous la présidence de Theodore Roosevelt que les États-Unis s'affirment sur la scène politique internationale en tant qu'acteur 
majeur. Sa vision réaliste des relations internationales, le concept d'équilibre des forces, sa politique d'expansionnisme impérialiste, la politique du Big Stick, le corollaire à la doctrine Monroe, son rôle de médiateur dans le conflit russo-japonais, sont autant de contributions majeures à la politique étrangère américaine $d u x^{\text {ème }}$ siècle, faisant de Theodore Roosevelt un président à la fois pragmatique et conscient des réalités de l'ordre mondial et de ses évolutions. Le droit des États-Unis d'intervenir préventivement, affirmé dans le corollaire à la doctrine Monroe, n'est pas sans annoncer la doctrine formulée par le président George W. Bush au lendemain des attentats du 11 septembre 2001. Theodore Roosevelt avait compris que des événements extérieurs pouvaient menacer la sécurité des États-Unis, et sa vision des relations internationales a considérablement influencé la politique extérieure de Franklin Delano Roosevelt et sa conception d'un système de sécurité collective au lendemain de la Seconde Guerre mondiale.

3 J. Simon ROFE (Université de Leicester) met ensuite en lumière l'importance de l'Advisory Committee for Problems in Foreign Relations (ACPFR) créé fin 1939 au sein du département d'État. Dirigé par le sous-secrétaire d'État Sumner Welles, le comité est alors chargé d'évaluer les conséquences du conflit mondial et d'émettre des propositions concernant les problèmes de reconstruction. Les réflexions du comité sur le désarmement, la formation d'une force de police internationale, la sécurité, la souveraineté nationale et même l'expansionnisme soviétique, influencent considérablement les activités de son successeur, l'Advisory Committee on Post-War Planning. Les travaux de l'ACPFR mettent en évidence le point de vue de l'administration Roosevelt sur l'ordre mondial d'après-guerre, dans le contexte de la " drôle de guerre » et des succès militaires de l'Axe en Europe. L'ACPFR envisage, dans l'après-guerre, un rôle important pour les États-Unis sur la scène internationale afin de promouvoir les intérêts américains de maintien de la sécurité nationale et internationale, et de la prospérité économique du pays. Simon Rofe insiste sur le dilemme auquel les membres de l'ACPFR sont confrontés : trouver des stratégies pour promouvoir les intérêts politiques et économiques des États-Unis dans le monde de l'après-guerre tout en affichant une neutralité défendue officiellement par l'administration Roosevelt et chère à l'opinion publique américaine.

Ruud V.A. JANSSENS (Université d'Amsterdam) s'intéresse ensuite aux politiques de transformation institutionnelle, économique et sociale durant l'occupation américaine du Japon. Des réformes structurelles profondes sont imposées pour éliminer le militarisme nippon et établir une tradition démocratique durable, parmi lesquelles figurent la mise en place d'une réforme agraire et l'instauration d'une démocratie parlementaire dès 1945. La nouvelle constitution, adoptée en mai 1947 par l'assemblée japonaise récemment constituée, introduit la notion de libertés individuelles et la nonbelligérance du Japon. Malgré les réticences de l'administration américaine, le général MacArthur insiste pour conserver le tenno (l'Empereur) en tant que garant de l'unité nationale, même si l'affirmation de la liberté de culte dans la nouvelle constitution met un terme au caractère sacré de la fonction impériale. Ruud JANSSENS rappelle que l'assentiment de la population est nécessaire pour assurer le succès des politiques d'occupation. Peu de réformes mises en place par le gouvernement militaire ont été révoquées après la fin de l'occupation en 1951. Alors que la guerre de Corée marque un tournant dans la guerre froide, les États-Unis soutiennent une politique de relance économique afin de faire de l'ancien ennemi une puissance régionale capable de s'ériger en rempart contre l'expansion communiste en Extrême-Orient. Le département 
de la Défense soutient la signature d'un traité de paix, le 8 septembre 1951, afin de sceller l'alliance nippo-américaine et la position stratégique des États-Unis en ExtrêmeOrient dans la guerre froide.

5 S'interrogeant sur les notions de continuité et d'analogie historique, Michaela HÖNICKE-MOORE (University of Iowa) évoque l'occupation américaine de l'Allemagne, présentée par l'administration Bush comme un modèle de réussite de nation building devant servir d'exemple à la reconstruction de l'Irak d'après Saddam Hussein. En 2003 paraît une étude menée par la Rand Corporation, America's Role in Nation-Building from Germany to Iraq qui influence les débats sur la politique étrangère américaine en Irak. Bien que l'étude insiste sur les spécificités propres au conflit mondial, elle conclut néanmoins que l'exemple de l'occupation américaine de l'Allemagne prouve que le transfert de démocratie est possible grâce à des moyens politiques et militaires. Selon Michaela Hönicke-Moore, les origines de la Seconde Guerre mondiale, les préparatifs de l'occupation ainsi que la nature des relations entre occupants et occupés diffèrent fondamentalement des conflits ultérieurs décrits dans l'étude. L'occupation de l'Allemagne ne saurait être érigée en modèle de transfert de démocratie. Michaela Hönicke-Moore distingue néanmoins trois éléments de continuité historique entre la Seconde Guerre mondiale, la guerre froide et la guerre contre le terrorisme : les leçons de Munich et la militarisation de la politique étrangère américaine, la lutte contre les totalitarismes et le rôle de l'idéologie dans le processus décisionnel, et la rupture avec les positions isolationnistes et non interventionnistes. Si l'occupation américaine de l'Allemagne a été un succès, c'est aussi et surtout parce que l'engagement américain ne s'est pas limité à renverser militairement le régime hitlérien. Les États-Unis ont affirmé leur vision pour le nouvel ordre mondial d'après-guerre, fondée sur le multilatéralisme, la défense des droits de l'homme, le libéralisme économique et la démocratie.

6 Lors de la deuxième session consacrée à l'institutionnalisation de l'exportation de la démocratie dans la guerre froide, Gérard BOSSUAT (Université de Cergy-Pontoise) offre une étude de cas de promotion de la démocratie dans la France de l'après-guerre. L'aide économique du plan Marshall s'accompagne, en effet, d'efforts de transformation économiques et politiques. Les États-Unis poursuivent une politique de construction économique européenne : les pays bénéficiaires du plan Marshall doivent s'unir pour assurer une gestion efficace de l'aide économique. Les réformes économiques, encouragées par l'Administration de Coopération Economique américaine (ECA), doivent entraîner des effets politiques et en définitive, permettre une stabilité et une paix durables sur le continent européen. Les archives révèlent que l'aide américaine s'accompagne d'une forme d'éducation aux méthodes modernes de gouvernement, les États-Unis encourageant notamment la suppression des barrières douanières, la liberté des échanges, et le retour à l'équilibre budgétaire et monétaire. Bien que celle-ci soit source d'inflation, les Américains laissent néanmoins la France utiliser la contre-valeur pour financer les investissements et la modernisation du pays. Si, en France, l'effort américain entraîne un regain de fierté nationale et une méfiance à l'égard de l'interdépendance transatlantique, les valeurs portées par le plan Marshall sont largement partagées par le milieu gouvernemental français.

7 Annick CIZEL (Université Sorbonne Nouvelle - Paris 3) s'intéresse au modèle de développement de l'Éthiopie, un pays africain indépendant, au sortir de la Seconde Guerre mondiale. Tributaire des rapports de forces internationaux, l'Éthiopie constitue alors une étude de cas d'«ingérence impérialiste» des États-Unis dans la Corne de 
l'Afrique, à l'invitation de l'empereur Haïlé Sélassié. Brandissant la menace d'un expansionnisme soviétique, les Américains tentent alors d'imposer leur présence et leur modèle de développement dans de nombreux pays africains et asiatiques en voie de décolonisation. Alors que les tensions est-ouest s'exacerbent, la position géostratégique de l'Éthiopie, entre Afrique et Moyen-Orient, place le régime autoritaire au cœur de toutes les attentions de l'administration Eisenhower. Érigé en rempart contre l'expansion communiste et le nationalisme égyptien tout à la fois, et en modèle pour les pays africains en voie de décolonisation, l'empire éthiopien connait en quelques années un important développement économique et, surtout, militaire. L'économie éthiopienne, marché stratégique pour l'industrie américaine, et en particulier l'aviation, bénéficie de capitaux américains qui contribuent au développement du pays. À l'heure où de nombreux pays afro-asiatiques proclament leur volonté d'émancipation, leur anticolonialisme et leur non-alignement, le penchant pro-américain de l'Éthiopie finit par nuire aux rapports diplomatiques entre Haïlé Sélassié et les pays arabes voisins, en particulier l'Égypte. Au début des années soixante, Gamal Abdel Nasser finance ainsi des mouvements séparatistes réclamant l'indépendance de la province de l'Érythrée, plongeant l'Éthiopie dans une guerre civile qui durera trente ans. C'est à cette époque que les Américains commencent à se désintéresser de cet État africain qui, au lendemain des décolonisations et face à la montée des tensions à Cuba et au Vietnam, ne constitue plus une priorité à leurs yeux.

Justine FAURE (IEP de Strasbourg) offre une perspective de la guerre froide culturelle en s'intéressant au rôle des échanges scientifiques dans la promotion de la démocratie. L'International Research and Exchanges Board (IREX), créé en 1968, devient l'une des principales organisations non gouvernementales responsable des échanges en sciences humaines et sociales entre les États-Unis et le bloc soviétique. Bien que l'engagement de la communauté scientifique et des fondations philanthropiques soit constant et leurs motivations diverses, les échanges scientifiques ont une dimension politique indéniable. Ils offrent en effet l'opportunité de combattre les préjugés, d'améliorer l'image des États-Unis, d'exposer les élites à des idées nouvelles, de former des experts et à terme d'affaiblir et de libéraliser les régimes communistes. Il est intéressant de constater, comme le souligne Justine Faure, que les acteurs des échanges universitaires n'utilisent pas les termes "démocratie » ni " démocratisation », préférant les termes «libéralisation » et « liberté ». Ils affichent ainsi une certaine prudence, par crainte de heurter leurs homologues derrière le Rideau de Fer et de compromettre la pérennité des échanges. Si l'objectif des échanges n'est pas officiellement l'exportation de la démocratie, ceux-ci permettent néanmoins la création de réseaux favorables aux ÉtatsUnis en Europe de l'Est. À cet égard, les échanges ont permis l'émergence d'une élite instruite, réformiste et pragmatique qui, après 1989, s'est montrée particulièrement active lors de la mise en place d'une économie de marché.

Évoquant l'interventionnisme des États-Unis au Nicaragua sous la présidence de Ronald Reagan, Stephen G. RABE (University of Texas) met en évidence les tensions existant entre la promotion de la démocratie et la lutte contre le communisme. En 1979, la dictature des Somoza, fervents anticommunistes soutenus par les États-Unis, est renversée par les Sandinistes qui installent un gouvernement d'extrême gauche. Bien que Washington n'ait pas d'intérêts stratégiques tangibles en Amérique centrale, l'enjeu reste la réaffirmation de l'hégémonie américaine sur le continent et la crédibilité des États-Unis sur la scène internationale. Au nom d'une croisade contre l'«Empire du Mal», le président Reagan soutient des opérations clandestines afin de 
déstabiliser le régime sandiniste, allant jusqu'à autoriser la CIA à former une unité paramilitaire nicaraguayenne, surnommée la Contra. En violation flagrante des lois nationales et internationales, des commandos américains mènent des opérations de sabotage dans les ports et les réservoirs pétroliers en 1983 et 1984. En 1986, la Cour internationale de justice condamne les États-Unis pour utilisation illégale de la force. La politique étrangère américaine au Nicaragua est par ailleurs menée au mépris de la constitution américaine et du pouvoir législatif, les États-Unis ayant secrètement vendu des armes à l'Iran afin de contourner l'amendement Boland et financer les Contra. L'élection en 1990 de l'opposante Violeta Barrios de Chamorro, avec le soutien des États-Unis, met un terme à la guérilla menée par les Contra, laissant un pays dévasté et épuisé par dix ans de violence et de conflits.

10 En s'intéressant au rôle actif du Congrès américain dans le processus de paix en BosnieHerzégovine, Maya KANDEL (IRSEM) offre la perspective d'un effort de reconstruction nationale post-guerre froide. La fin de la guerre froide, l'échec de l'intervention américaine en Somalie en 1993, et un fort sentiment anti-Clinton porté par un Congrès à majorité républicaine à partir de 1995 incitent les parlementaires à s'investir davantage dans la politique extérieure américaine. C'est après les révélations des massacres perpétrés durant l'été 1992 que l'opinion publique américaine et le Congrès s'invitent dans le débat sur la Bosnie. Dans ce conflit en ex-Yougoslavie s'opposent un désir de paix des Européens et un désir de justice porté par le Congrès américain. Alors que les Européens envisagent le retrait de leurs contingents militaires afin de ramener la stabilité sur le continent, le Congrès américain soutient le droit des musulmans bosniaques à l'autodéfense et leur droit de porter des armes. Durant l'été 1995, dans un contexte de campagne électorale, le Congrès vote la levée unilatérale de l'embargo sur les armes à destination de la Bosnie, et ce en dépit de l'opposition du président Clinton, des Alliés et de la Russie. Les accords de Dayton conclus en novembre 1995 prévoient la mise en place d'un programme de soutien logistique et de formation des forces armées croates et des soldats musulmans bosniaques afin de stabiliser les forces militaires en présence et de refouler les Serbes. La dimension militaire de la mission américaine en Bosnie dans le cadre de la force internationale IFOR démontre d'une part, l'influence du Pentagone et $d u$ Congrès républicain et, d'autre part, les faiblesses d'un président Clinton dont la politique étrangère est décrédibilisée. Les accords de Dayton démontrent également la méfiance des Américains envers l'ONU et l'évolution stratégique de l'OTAN à cette période en tant qu'organisation chargée du maintien de la paix.

11 En conclusion à la journée, Tony SMITH (Tufts University) évoque la permanence de la tradition wilsonienne de promotion de la démocratie sur la scène internationale à travers l'histoire de la politique étrangère américaine. La période " pré-classique » voit la conceptualisation de la notion de démocratie mais ne s'accompagne pas d'un effort d'exportation, la jeune république étant consciente des limites de son pouvoir. La période dite "classique » est marquée par l'émergence de l'internationalisme libéral, centrée autour de quatre aspects essentiels: la gouvernance démocratique, l'interdépendance économique, le multilatéralisme et le leadership américain. La promotion de la démocratie semble incarner le meilleur espoir pour la stabilité et la paix mondiale. Pour Woodrow Wilson, la solution réside dans la sécurité collective garantie par des États démocratiques réunis au sein d'une organisation multilatérale, la Société des Nations. Ce n'est qu'à la fin de la Seconde Guerre mondiale que les idées de Woodrow Wilson seront institutionnalisées, avec notamment les accords de Bretton 
Woods, l'Organisation des Nations Unies, et la construction européenne. La chute du bloc soviétique marquera la victoire de l'hégémonie américaine et le triomphe du wilsonisme en tant qu'idéologie. Si durant la guerre froide, note Tony Smith, l'internationalisme libéral manque de fondement théorique, à la différence du marxisme-léninisme, les années 1990 voient l'émergence du concept de Democratic Peace Theory. La conviction que le modèle américain de démocratie libérale est moralement supérieur et doit être exporté est reprise par le président George W. Bush, qui défend le principe de "guerre préventive » et de "guerre juste » menée contre des régimes non démocratiques. La doctrine Bush est ainsi née de la vision wilsonienne de promotion de la démocratie. Selon Tony Smith, le plus grand succès de l'histoire de la politique étrangère américaine est la démocratisation de l'Allemagne et du Japon. Paradoxalement, ce que l'internationalisme démocratique libéral a créé en Allemagne et au Japon au lendemain de la Seconde Guerre mondiale s'est soldé par un échec en Irak et en Afghanistan.

Dans un contexte de crise économique mondiale, de bouleversement majeur des équilibres dans le monde arabe, de bilan de l'intervention américaine en Irak et en Afghanistan, les réflexions proposées durant cette journée sur les transitions démocratiques, les reconstructions post-conflit, les politiques de transformation et la souveraineté populaire se sont révélées à la fois nécessaires et éclairantes. Cette journée d'études sera suivie d'une nouvelle rencontre les 18-19 octobre 2012 qui offrira une perspective post-guerre froide, de la guerre des Balkans aux révolutions arabes.

\section{INDEX}

Thèmes : Actualité de la recherche

\section{AUTEUR}

\section{MYRIAM CHASSERIEAU}

Université Aix-Marseille 